\title{
The epidermal growth factor receptor (EGF-R) is present on the basolateral, but not the apical, surface of enterocytes in the human gastrointestinal tract
}

\author{
R J Playford, A M Hanby, S Gschmeissner, L P Peiffer, N A Wright, T McGarrity
}

Department of

Medicine and

Therapeutics,

University of

Leicester, Leicester

R J Playford

Departments of Histopathology

A M Hanby

N A Wright

and Electron

Microscopy

S Gschmeissner

Imperial Cancer Research Fund, London

Division of

Gastroenterology,

Hershey Medical

Center, Hershey, USA

L P Peiffer

T McGarrity

Correspondence to: Professor R Playford, Department of

Gastroenterology, Leicester General Hospital NHS Trust, Gwendolen Road, Leicester LE5 4PW.

Accepted for publication 13 February 1996

\begin{abstract}
Background-While it is clear that luminal epidermal growth factor (EGF) stimulates repair of the damaged bowel, its significance in maintaining normal gut growth remains uncertain. If $\mathbf{E G F}$ is important in maintaining normal gut growth, the EGF receptor (EGF-R) should be present on the apical (luminal) surface in addition to the basolateral surface. Aims/Subjects/Methods-This study examined the distribution of the EGF-R in the epithelium throughout the human gastrointestinal tract using immunohistochemistry, electron microscopy, and western blotting of brush border preparations.

Results-Immunostaining of the oesophagus showed circumferential EGF-R positivity in the cells of the basal portions of the stratified squamous epithelium but surface cells were EGF-R negative. In the normal stomach, small intestine, and colon, immunostaining localised the receptor to the basolateral surface with the apical membranes being consistently negative. EGF-R positivity within the small intestine appeared to be almost entirely restricted to the proliferative (crypt) region. Western blotting demonstrated a $170 \mathrm{kDa}$ protein in whole tissue homogenates but not in the brush border vesicle preparations.

Conclusions-As the EGF-R is located only on the basolateral surfaces in the normal adult gastrointestinal tract, the major role of luminal EGF is probably to stimulate repair rather than to maintain normal gut growth.

(Gut 1996; 39: 262-266)
\end{abstract}

Keywords: cell proliferation, gut growth, localisation, immunohistochemistry, electron microscopy, growth factors.

Epidermal growth factor (EGF) is a potent mitogen, which is continuously secreted into the gut lumen by the salivary glands and the Brunner's glands of the duodenum. ${ }^{1}$ Many studies have shown that EGF stimulates proliferation of various cell lines in vitro and also stimulates gastrointestinal growth in whole animals when given via the systemic circulation. ${ }^{2}$ It is also generally accepted that luminal EGF can stimulate repair of the damaged bowel. ${ }^{3}$ There is continuing controversy, however, regarding the role of luminal EGF in maintaining normal gut growth in the adult human intestine. If EGF is important in maintaining gut growth, one would expect the EGF receptors to be present on the apical (luminal) surfaces of the gastrointestinal epithelium. We therefore examined the distribution of the EGF receptor (EGF-R) in the normal human adult gastrointestinal tract using a combination of immunohistochemistry, electron microscopy, and western blotting of brush border preparations.

\section{Methods}

\section{Ethical approval}

This project had local ethical approval and all subjects gave informed written consent. All biopsy specimens were obtained during routine endoscopy performed for clinical reasons. Immunohistochemical and electron microscopy analyses were performed at the Imperial Cancer Research Fund, London and the western blot analyses performed at The Pennsylvania State University, USA.

\section{Patients and collection of samples}

Fresh endoscopic biopsy specimens were obtained from the oesophagus, stomach, small intestine, and colon of 20 histologically normal subjects. Patients donating upper gastrointestinal specimens all had a final diagnosis of nonulcer dyspepsia and patients donating colonic specimens all had a final diagnosis of the irritable bowel syndrome. Biopsy forceps FB 24Q were used to collect oesophagus, gastric, and duodenal specimens and FG13U forceps used to collect colonic and ileal specimens (both forceps obtained from Olympus Corporation, Lake Success, NY). Ileal specimens were obtained during colonoscopy by cannulation of the ileocaecal valve. The average weight of upper intestinal specimens was $9 \pm 1 \mathrm{mg}$ and of colonic specimens was $11 \pm 1 \mathrm{mg}$. Samples for immunohistochemistry were fixed in formal saline, processed routinely, and embedded in paraffin wax in the conventional manner. Fresh sections were cut and stained with haematoxylin and eosin to establish that no histological abnormality was present. Samples for western blotting were snap frozen in liquid nitrogen and stored at $-70^{\circ} \mathrm{C}$ until assay. 


\section{Immunohistochemistry for $E G F-R$}

Mouse antihuman EGF- $R$ monoclonal antibody was raised using purified human EGF-R, derived from A-431 cells, as the antigen (Triton Diagnostics, Alameda, CA, USA). Freshly cut sections were dewaxed and incubated in $100 \%$ alcohol. Endogenous peroxidase activity was blocked using a 10 minute incubation period in methanol containing $0.02 \%$ hydrogen peroxide ( $\mathrm{vol} / \mathrm{vol})$. Sections were washed in phosphate buffered saline (PBS) and masked antigenic sites revealed by incubating in PBS solution containing 0.04\% Nagarase (Cat no P-8038, Sigma UK) for 30 minutes. Sections were incubated in primary mouse antihuman EGF-R monoclonal antibody at a final dilution of 1:25 for 35 minutes, washed in PBS, and incubated in biotinylated rabbit antimouse antibody (Dako E354) at a final dilution of 1:500 to which 1:25 normal human serum had been added. Sections were again washed in PBS followed by incubation in streptavidin-peroxidase at 1:500 for 30 minutes. A brown reaction product was obtained using a peroxidase substrate (diaminobenzidine, PBS, in addition to $0.3 \%$ hydrogen peroxide). As a negative control, the primary antibody was omitted from the procedure. In addition, the specificity of the staining was confirmed by taking parallel sections and incubating in the presence of excess recombinant EGF-R.

Rabbit antihuman EGF-R polyclonal antibody $12 E$ was produced by Gullick and coworkers using a short synthetic peptide sequence corresponding to residues $1164-1176$ of the intracytoplasmic region of the EGF-R as the antigen. ${ }^{4}$ Sections were prepared as described above and incubated with the primary antibody at a final concentration of $1 / 40$. The second antibody reaction consisted of incubating sections in biotinylated swine-antirabbit antibody at a final concentration of 1:500 to which 1:25 normal human serum had been added. As a negative control, the primary antibody was omitted from the procedure. In addition, the specificity of the staining was confirmed by taking parallel sections and incubating in the presence of excess recombinant EGF-R.

\section{Electron microscopy}

Fresh tissues were fixed with $4 \%$ paraformaldehyde in $0.1 \mathrm{M}$ Sorensens phosphate buffer ( $\mathrm{pH} \mathrm{7.4)}$ for eight hours at $4^{\circ} \mathrm{C}$. After fixation, the tissues were treated with $0.5 \mathrm{M}$ $\mathrm{NH}_{4} \mathrm{Cl}$ in phosphate buffer for four hours and washed in buffer overnight. The tissues were dehydrated in a graded series of methanol concentrations $(50,70,80,90 \%$ and absolute methanol, each for 10 minutes) at progressively lower temperatures and infiltrated with Lowicryl HM 20 at $-35^{\circ} \mathrm{C}$. The resin was polymerised by ultraviolet light for 48 hours at $-50^{\circ} \mathrm{C}$.

For immunogold studies, ultrathin sections were mounted on carbon coated grids and labelled as follows. After five minutes on a drop of PBS and one hour preincubation on $10 \%$ normal goat serum, $5 \%$ bovine serum albumin (BSA), and 5\% ovalbumin in PBS, the grids were transferred onto drops of rabbit antihuman EGF-R antiserum, 12E (1:40 dilution in PBS containing $0.5 \%$ BSA, $0.5 \%$ ovalbumin, and $1 \%$ normal goat serum) for eight hours at $4^{\circ} \mathrm{C}$. Sections were washed three times over a 15 minute period with PBS containing 0.5\% BSA, $0.5 \%$ ovalbumin, and $1 \%$ normal goat serum followed by incubation with immunogold conjugates (1:100 goat antirabbit IgG, $5 \mathrm{~nm}$ gold) for two hours. After rinsing in PBS ( $3 \times$ five minutes) and distilled water ( $3 \times$ five minutes), the sections were silver enhanced for four minutes at room temperature using an Intense M Silver Enhancement Kit (Amersham, UK) before contrasting with uranyl acetate and lead citrate. Sections were then examined using a Zeiss $10 \mathrm{C}$ electron microscope. As a negative control, the primary antibody was omitted from the procedure.

\section{Western blotting}

Background to method - to determine if the apical membranes contained EGF-R, purified brush border vesicles were prepared and probed for the presence of EGF-R protein using western blotting. The quality of the brush border preparations was determined by following changes in the specific activity of alkaline phosphatase activity, whose expression is restricted to the apical membranes. ${ }^{5}$ Two rabbit polyclonal antibodies were used for these studies; 12E, which had also been used for the immunohistochemical localisation studies described above and a further affinity purified rabbit-antihuman EGF-R raised against a synthetic peptide corresponding to residues 1005 to 1016 of the human EGF receptor protein (EGFR-1005, Santa Cruz Biotechnology, Santa Cruz, CA).

Preparation of brush border vesicles-brush border preparations were made from duodenal and ileal tissue using the protocol described by Simpson, ${ }^{6}$ which is a modification of the original technique of Kessler. ${ }^{5}$ In these methods, the microvesicle samples are purified from potentially contaminating intracellular organelles by incorporating a centrifugation step in which $\mathrm{MgCl}_{2}$ had been pre-added to the diluent (which causes the organelles, but not the brush border vesicles, to precipitate). This protocol produces apical membrane preparations that are essentially free from contaminating organelles as judged by electron microscopy and marker enzymes. ${ }^{56}$ Aliquots of the initial tissue homogenate and the final microvesicle preparations were assayed for total protein ${ }^{7}$ and alkaline phosphatase activity (Sigma alkaline phosphatase assay kit, St Louis, USA).

Western blotting - this was performed using one dimensional SDS-PAGE electrophoresis. Eighty $\mu \mathrm{g}$ of sample protein were loaded into each lane of a $7 \cdot 5 \%$ SDS-PAGE gel. Samples consisted of whole tissue homogenate, microvesicle preparations, and prestained molecular weight markers. After electrophoresis, the proteins were electroblotted onto nitrocellulose paper and stained with Ponceau $S$ to confirm complete transfer and loading 
efficiency. The nitrocellulose filters were then destained prior to incubation in a blocking solution for 30 minutes ( $5 \%$ non-fat dry milk, $0.5 \mathrm{M} \mathrm{NaCl}, 0.02 \mathrm{M}$ TRIS pH $7.4,0.1 \%$ TWEEN 20). Filters were then incubated with primary antibodies (E12 or EGF-R 10050, each at $5 \mu \mathrm{g} / \mathrm{ml}$ ) for 45 minutes and washed three times with TBS (containing 0.05\% TWEEN 20). Blots were then incubated with horseradish peroxidase conjugated goat antirabbit antibody diluted $1: 1000$ in 5\% nonfat dry milk for 30 minutes. Filters were then washed three times in TBS, $0.05 \%$ TWEEN

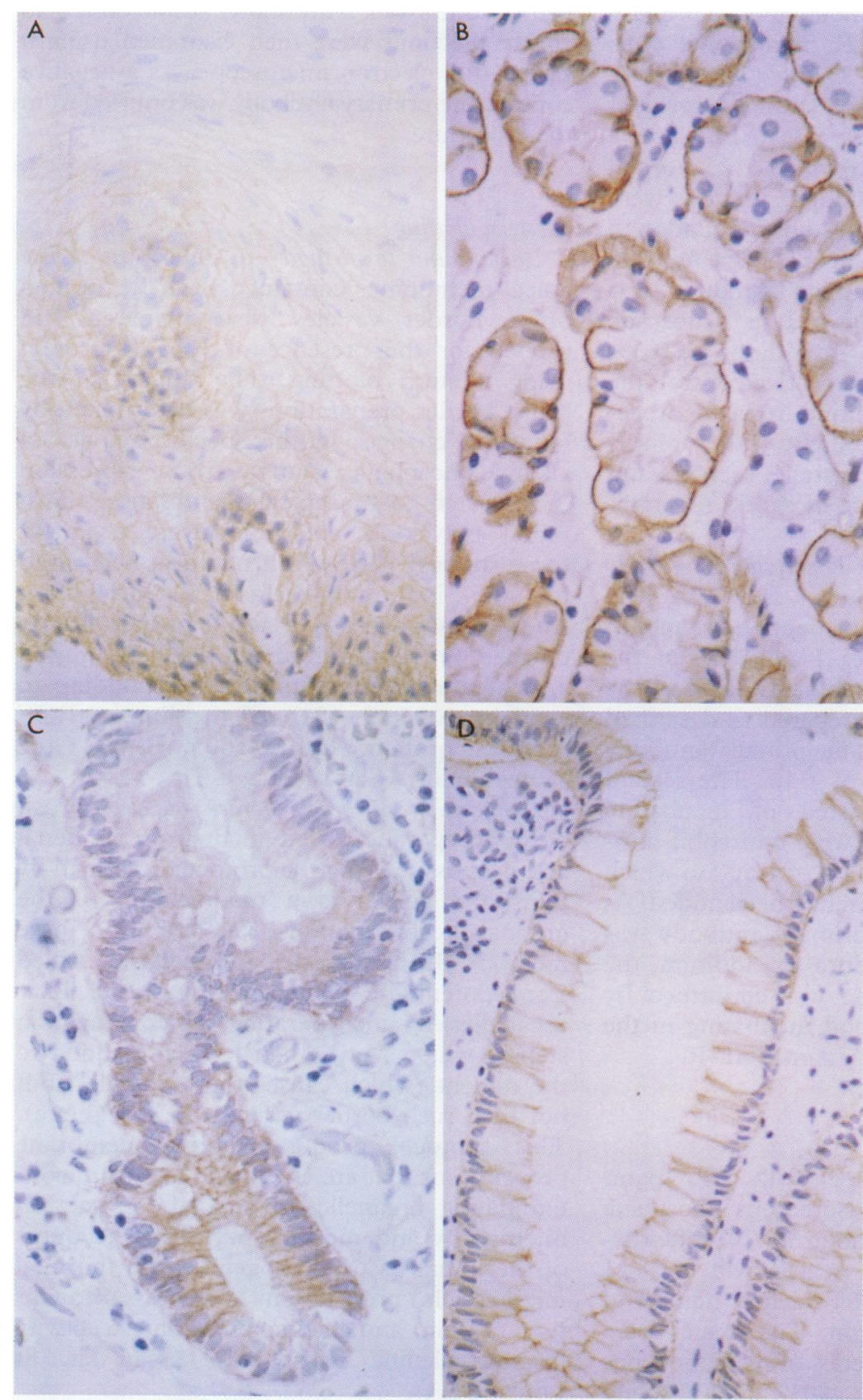

Figure 1: Immunohistochemical localisation of the EGF-R using rabbit antihuman polyclonal antibody $12 E$. EGF-R positivity was visualised using $D A B$, giving a brown reaction product. In the oesophagus, weak EGF-R positivity is seen circumferentially in cells of the basal region of the stratified squamous epithelium but EGF-R positivity disappears as the cells approach the luminal surface $(A$, original magnification $\times 400)$. In the stomach, EGF-R positivity was seen on the basolateral surfaces on surface, parietal, and mucus neck cells with particularly strong staining on the parietal cells (B, original magnification $\times 400)$. In the small intestine, $E G F-R$ positivity was restricted to the basolateral membranes of the proliferative (crypt) region and the goblet cells of the villi, with little or no positivity on the villus enterocytes (C, original magnification $\times 250)$. In the colon, EGF-R positivity was seen on the basolateral surfaces of cells in all regions of the crypts with relatively weak staining of the basolateral membranes of the surface colonocytes. $(D$, original magnification $\times 400$.)
20 followed by one wash in TBS (each wash for five minutes). The presence of the EGF-R proteins was then visualised using the ECL (chemiluminescence) system (Amersham, UK) by incubating in the ECL reagents for one minute as recommended by the manufacturer. All assays were performed in quadruplicate.

To determine the specificity of the reaction, western blots were performed in which the primary antibody had been pre-incubated in excess recombinant EGF-R (kindly donated by W Gullick, ICRF).

\section{Results}

Immunohistochemistry and electron microscopy In the oesophagus, weak membrane localised EGF-R positivity was seen circumferentially in the deeper portions of the stratified squamous epithelium but surface cells were negative (Fig 1A). The gastric body and antrum epithelium showed EGF-R-like immunoreactivity in the basolateral membranes with particularly strong staining of the parietal cells. Surface membranes were consistently negative (Fig 1B). Duodenal and ileal tissue showed EGF-R-like positivity on the basolateral surfaces of the crypt regions (Fig 1C) and on the goblet cells of the villi, however, the enterocytes on the villi appeared to be EGF-R negative. Colonic mucosa showed EGF-R-like positivity in a basolateral distribution in all regions of the crypts (Fig 1D). Distribution of EGF-R positivity visualised using electron microscopy gave similar results (Fig 2). Therefore, the apical/surface membranes abutting the intestinal lumen were consistently negative throughout the gastrointestinal tract. The negative control and slides in which excess recombinant EGF-R had been added showed no staining, confirming the specificity of the reaction.

\section{Western blotting}

On each occasion, brush border preparations showed a pronounced increase in alkaline phosphatase activity (typical specific activity of alkaline phosphatase in ileal whole tissue homogenate was $0.1 \mathrm{U} / \mathrm{mg}$ protein compared with $0.9 \mathrm{U} / \mathrm{mg}$ protein in brush border preparations). The major band seen in duodenal and ileal whole tissue homogenates had an apparent molecular weight of approximately $170 \mathrm{kDa}$, with some fainter smaller bands (with apparent molecular weights of approximately 150 and $100 \mathrm{kDa}$ ) being inconsistently seen in whole tissue homogenates of ileal preparations. Brush border vesicle preparations from these tissues were consistently negative (Fig 3). Importantly, no bands were seen in any of the lanes in parallel western blots in which the primary antibody had been preincubated in excess recombinant EGF-R.

\section{Discussion}

Using a variety of different techniques, we have examined the distribution of the EGF-R in the human gastrointestinal tract. All results 


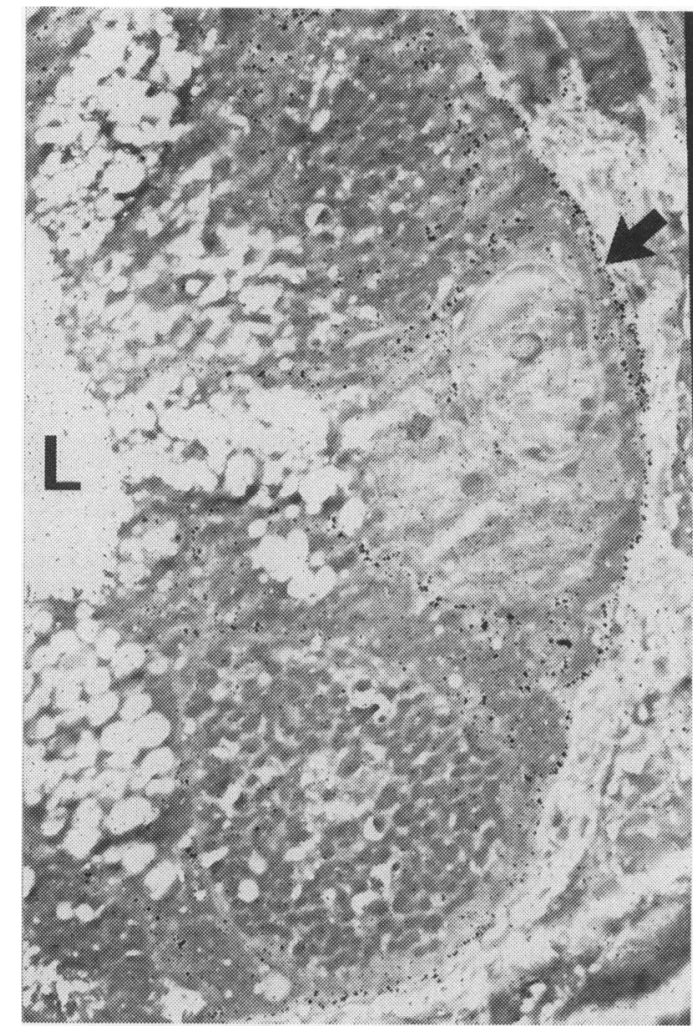

Figure 2: Electron microscopy of gastric body showing $E G F-R$ positivity (visualised using gold tagged antibody) is present on the basolateral but not the apical (luminal, $L$ ) membranes of gastric glands. This is particularly well seen on the arrowed cell. Original magnification $\times 6000$.

suggest that, in the normal human adult gut, the apical/surface membranes abutting the intestinal lumen do not express EGF-R.

We used two different antibodies (one monoclonal and one polyclonal) to examine the distribution of the EGF-R by immunohistochemistry to decrease the possibility of false EGF-R-like positivity. For all regions of the gastrointestinal tract, results were consistent between the two. The major form of EGF$\mathbf{R}$ found in tissue homogenates of duodenum and ileum had an apparent molecular weight of about $170 \mathrm{kDa}$, although fainter bands were sometimes seen at approximately 150 and 100 $\mathrm{kDa}$, probably representing partial degradation products of the EGF-R.

EGF is a 53 amino acid peptide (molecular weight 6400), which is secreted into the gastrointestinal lumen from the salivary glands and Brunner's glands of the duodenum. ${ }^{1}$ Gastric juice concentrations of EGF are about $500 \mathrm{ng} / 1,8$ sufficient to stimulate growth of epithelial cells in vitro. The concentration of luminal EGF in the small and large intestine is less certain, but probably varies according to the presence or absence of ingested proteins as EGF is susceptible to digestion by pancreatic proteases. ${ }^{9}$ The sequence and structure of the EGF-R is well characterised and consists of an intracellular domain (with tyrosine kinase activity), a single transmembrane region, and an extracellular domain with a single ligand binding site. Although many studies have shown that EGF is a potent mitogen for gastrointestinal tissue in vitro and when given systemically in vivo, ${ }^{2}$ there is continuing debate as to its role

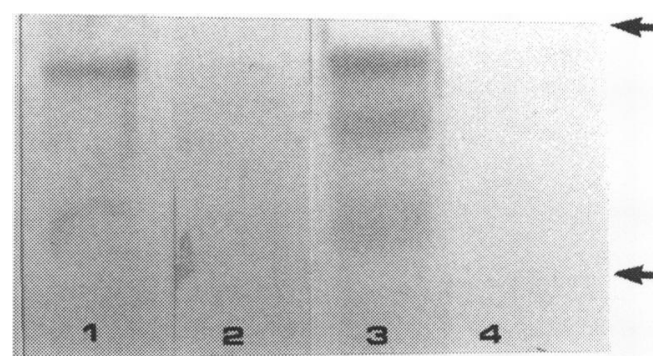

Figure 3: Western blots of tissue homogenates and microvillus preparations using rabbit antihuman EGF-R antibody $12 E$. Eighty micrograms of protein were loaded per lane in the following distribution; whole tissue homogenate (lane 1) and microvillus preparation of duodenum (lane 2), and whole tissue homogenate lane 3) and microvillus preparations of ileum (lane 4). Arrows show the position of protein markers of molecular weights 220 and $90 \mathrm{kDa}$. Bands corresponding to full length $E G F-R$ are seen in whole tissue homogenates of duodenum and ileum, but not in the microvillus preparations suggesting that the distribution of EGF-R is limited to basolateral membranes. Note that ileal whole tissue homogenates (lane 3) also showed inconsistent bands of approximately 150 and $100 \mathrm{kDa}$, probably corresponding to a partial degraded form of EGF-R. No bands were seen in western blots performed in parallel in which the antibody had been pre-incubated with recombinant $E G F-R$.

in the normal gastrointestinal tract. If luminal EGF is important in maintaining normal gastrointestinal growth (or influencing gastric acid secretion, or both), one would expect the EGF-R to be present on the apical (luminal) membranes as the tight junctions of the intestinal epithelium probably prevents paracellular translocation of the peptide under normal circumstances.

Most of the previous studies in this area have examined the distribution of the EGF-R in animal models, particularly the rat. A paper by Thompson and coworkers suggests that, in the adult rat, the EGF-R is restricted to the basolateral membranes. ${ }^{10}$ However, species differences may exist as Kelly and coworkers reported that EGF-Rs are present on the apical membranes of the pig intestine (using the technique of autoradiographic localisation ${ }^{11}$ ). There have been few equivalent studies performed in humans and these have mainly focused on the distribution of the EGF-R in the oesophagus and stomach. Using immunohistochemical staining, Jankowski and coworkers reported EGF-R-like positivity in a circumferential distribution in the basal portion of the stratified squamous epithelium of the oesophagus, ${ }^{12}$ in keeping with our results. However, in contrast with our findings, they found that the EGF-R was present on the apical and basolateral membranes of the normal human stomach. ${ }^{13} \mathrm{~A}$ similar apical distribution has been also reported in the adult stomach and colon by Hormi and Lehy. ${ }^{14}$ Reasons for these differences are unclear, although the methods used by both groups were restricted to immunohistochemical localisation and the antibodies used by each group (Ab- 1 by Jankowski, and Ab- 4 by Hormi and Lehy, both monoclonal IgG's obtained from Oncogene Science, Manhaset, NY, USA) were different from the two used in this study. A different monoclonal antibody (528IgG) was used by Mori and coworkers to examine the 
distribution of the EGF-R in the human stomach using the technique of electron microscopy. They reported that the EGF-R was restricted to the basolateral membranes of parietal cells, ${ }^{15}$ in keeping with our findings. However, caution has to be shown in interpreting the results from this study as the 'apparently normal gastric fragments' were obtained from patients having gastric resections for gastric carcinoma. It was because of these apparently conflicting results that, in contrast to all of these other studies, we decided to use several different techniques and antibodies (both monoclonal and polyclonal) to examine the distribution of the EGF-R in detail.

Our confirmation that the EGF-R positivity was restricted to basolateral membranes suggests that the role of luminal EGF in the adult gut is to act as a 'luminal surveillance peptide'; readily available to stimulate repair at sites of injury (when it can reach its receptor), but of comparatively minor importance in maintaining gut growth. ${ }^{16}$ However, it is important to note that the EGF-R also binds transforming growth factor $\alpha$, amphiregulin, and heparin binding EGF. Our findings that the EGF-R is present on the basolateral surfaces of the parietal cells of the stomach and the proliferative (crypt) regions of the small and large intestine suggests that one or more of these other ligands may be important in controlling acid secretion and proliferation of the gastrointestinal tract.

It will be of interest to extend these studies to examine the distribution of the EGF-R in the neonatal or adult damaged bowel as animal studies suggest that, in these conditions, luminal EGF is capable of stimulating gut growth and repair. This may be because of an altered distribution of the EGF-R, or alternatively, that the increased permeability of the neonatal or damaged bowel allows luminal EGF to reach its receptor on the basolateral membranes.
We thank the Medical Research Council and the Wellcome Trust for funding.

1 Heitz PU, Kasper M, Van Norden S, Polak JM, Gregory H, Pearse AGE. Immunohistochemical localisation of urogastrone to human duodenal and submaxillary glands. Gut 1978 19: 408-13.

2 Goodlad RA, Wilson TJG, Lenton W, Gregory H, McClagh $\mathrm{KG}$, Wright NA. Intravenous but not intragastric urogastrone (EGF) is trophic to the intestine of parenterally fed trone (EGF) is trophic to the

3 Itoh M, Imai S, Kawai T, Katsumi K, Yokochi K, Takeuchi T. Protection of gastric mucosa against ethanol injury by
toh intragastric bolus administration of epidermal growth factor combined with hydroxypropylcellulose. $\mathcal{f}$ Clin Gastroenterol 1992; 14: S127-30.

4 Gullick WJ, Hughes CM, Mellon K, Neal DE, Lemoine NR. Immunohistochemical detection of the epidermal growth factor receptor in parrafin-embedded tissue. F Pathol 1991; 164: 284-9.

5 Kessler M, Acuto O, Storelli C, Murer H, Muller M, Semenza G. A modified procedure for the rapid preparation of efficiently transporting vesicles from small intestine tion of efficiently transporting vesicles from small intestine
brush border membranes. Biochem Biophys Acta 1978; 506: $136-54$.

6 Simpson RJ, Peters TJ. Studies of $\mathrm{Fe}^{3+}$ transport across isolated intestinal brush border membrane of the mouse. Biochem Biophys Acta 1984; 772: 220-6.

7 Schacterle G, Pollack R. A simplified method for the quantitative assay of small amounts of protein in biological material. Anal Biochem 1973; 51: 654-5.

8 Playford RJ. Marchbank T, Calam J, Hansen FH. EGF is digested to smaller, less active, forms in acidic gastric juice. Gastroenterology 1995; 108: 92-101.

9 Playford RJ, Watanaba P, Woodman AC, Deprez PH, Calam J. Effect of luminal growth factor preservation on intestinal growth. Lancet 1993; 341: 843-8.

10 Thompson JF, Van Den Berg M, Stokkers PCF. Developmental regulation of epidermal growth factor Developmental regulation of epidermal growth factor
receptor kinase in rat intestine. Gastroenterology 1994; 107: receptor

11 Kelly D, McFadyen M, King TP, Morgan PJ. Characterisation and localisation of the epidermal growth factor receptor in the jejunum of the neonatal and weaned pig. Reprod Fertil Dev 1992; 4: 183-9.

12 Jankowski J, Murphy S, Coghill G, Grant A, Wormsley KG, Sanders DSA, et al Epidermal growth-factor receptors in the oesophagus. Gut 1992; 33: 439-43.

13 Jankowski J, Al-Rawi HJ, Johnston DA, Hopwood D, Filipe MI, Coghill G, et al. Growth regulatory peptides in gastric mucosa. Clin Sci 1992; 82: 581-7.

14 Hormi K, Lehy T. Developmental expression of transforming growth factor $\alpha$ and epidermal growth factor receptor proteins in the human pancreas and digestive tract. Cell Tissue Res 1994; 278: 439-50

15 Mori S, Morishita Y, Sakai K, Kurimoto S, Okamoto M, Kawamoto T, et al. Electron microscopy evidence for epidermal growth factor receptor (EGF-R) like immunoreactivity associated with the basolateral surface of gastric pariental cells. Acta Pathol fpn 1987; 37: 1909-17.

16 Playford RJ. The role of peptides in mucosal integrity and repair. Gut 1995; 37: 595-7. 